\title{
ÍNDICE DE GENERACIÓN DE ESCOMBROS PRODUCIDOS EN LA CONSTRUCCIÓN DE VIVIENDA EN LA ZONA URBANA DE SINCELEJO, SUCRE, COLOMBIA.
}

\author{
INDEX OF RUBBLE GENERATION PRODUCED IN THE BUILDING URBAN AREA \\ OF SINCELEJO, SUCRE, COLOMBIA.
}

\author{
MARÍA C MONROY P, M.Sc.
}

Universidad de Sucre, Facultad de Ingeniería, Programa de Ingeniería Civil.

\section{Key words:}

Construcción, escombros, índice de generación de escombros,

Sincelejo,

Sucre.

\section{Abstract}

This paper analyzes the generation of debris in housing construction in Sincelejo city, Sucre, Colombia, assembling the strata one, two and three in a group and strata four, five and six in another one, According to the permits granted by the competent authority. The debris was ranked cement, geotextile, bricks, pipes, rod and wire. The residues were weighed and the calculation was based on volume and density of each proceeded to establish the index of residues or debris. the waste generation rate for Group 1 was $0.017 \mathrm{~m}^{3} / \mathrm{m}^{2}$, while Group 2 was $0.014 \mathrm{~m}^{3}$ / $\mathrm{m}^{2}$, there is no significant difference when comparing the two groups $(p<0.05)$; Consulted bibliography shows that the index $\mathrm{d}$ generation of construction waste can be between 0.050 to $0.178 \mathrm{~m}^{3} / \mathrm{m}^{2}$, as found in this study is lower between 10 and $34 \%$.

\section{Palabras Clave:}

Construction, debris management, waste generation rate, Sincelejo,

Sucre.
INFORMACIÓN Recibido: 02-07-2015: Aceptado: 13-11-2015. Correspondencia autor: mariacmonroy2007@hotmail.com

\section{Resumen}

El presente trabajo analiza la generación de escombros en la construcción de vivienda en la ciudad de Sincelejo, Sucre, Colombia, reuniendo los estratos uno, dos y tres en un grupo y los estratos cuatro, cinco y seis, según las licencias de construcción otorgadas por la autoridad competente. Los escombros fueron clasificados en cemento, geotextil, ladrillos, tuberías, varilla y alambre. Los residuos fueron pesados y se les cálculo el volumen y con base en la densidad de cada uno de ellos se procedió a establecer el índice de generación de residuos o escombros. El índice de generación de residuos para el Grupo 1 fue de $0,017 \mathrm{~m}^{3} / \mathrm{m}^{2}$, mientras que para el Grupo 2 fue de $0,014 \mathrm{~m}^{3} / \mathrm{m}^{2}$, no existe diferencia significativa al comparar los dos grupos $(p<0,05)$; bibliografía consultada muestra que el índice $d$ generación de residuos en obras de construcción puede estar entre 0,050-0,178 $\mathrm{m}^{3} / \mathrm{m}^{2}$, lo hallado en este trabajo es menor entre 10 y $34 \%$. 


\section{Introducción}

Los residuos sólidos o escombros producidos por la construcción urbana presentan bajo riesgo a la salud humana y al ambiente, comparándolos con los residuos sólidos de otra naturaleza que se generan en las urbes (GARCÍA-DONAS y AINCHIL LAVÍN, 2009). No obstante, los efectos de la construcción en el medio ambiente son altamente negativos; de acuerdo con ARENAS CABELLO (2007), la mitad de los materiales empleados en el total de la industria de la Construcción proceden de la corteza terrestre, siendo el sector el responsable del $50 \%$ de los recursos naturales empleados, del $40 \%$ de la energía consumida (incluyendo la energía en uso) y del $50 \%$ del total de los residuos generados.

En Sincelejo se observa un alto consumo de materiales de construcción por el acelerado incremento de la industria, generando gran cantidad de escombros con disposición inadecuada cerca a los sitios de generación, lugares no autorizados, arroyos, con invasión del espacio público y privado, generando contaminación e incrementando la sobreexplotación de recursos naturales y materiales pétreos de los entornos naturales, causando un agotamiento acelerado de las reservas de áridos provenientes tanto de los cauces de los ríos como de las canteras. Se evidencia ausencia gestión y disposición de estos materiales asociada a los grandes volúmenes generados, a los altos costos de transporte y al gran espacio requerido para su disposición final (SERRANO y FERREIRA, 2009).

Aspectos antes señalados muestran que se debe determinar la cantidad y el tipo de residuos sólidos producidos, además de realizar una adecuada gestión de los escombros que permita conocer de forma investigativa su producción en volumen y composición, igualmente, como insumo derivado de este estudio se ofrece un referente investigativo que permitirá que las autoridades competentes integren el manejo, reutilización y disposición de los residuos sólidos en mención.

\section{Materiales y método}

Área de estudio: Sincelejo, Sucre $\left(9^{\circ} 18^{\prime} \mathrm{N}\right.$ y $\left.75^{\circ} 23^{\circ} \mathrm{O}\right)$. En la Fig. 1 se muestran los sectores que identificados como estratos fueron motivo de evaluación.

Muestreos: Se llevaron a cabo durante los meses de enero a abril de 2012. El total de construcciones evaluadas se fundamentó en el número de licencias de construcción otorgadas en el año 2011 y que estaban en ejecución, según datos del ICER (2012) y fueron 186, de las cuales 73 estaban en estratos del 1 al 3 que se identifica como Grupo 1, y las restantes 113 se ubicaron

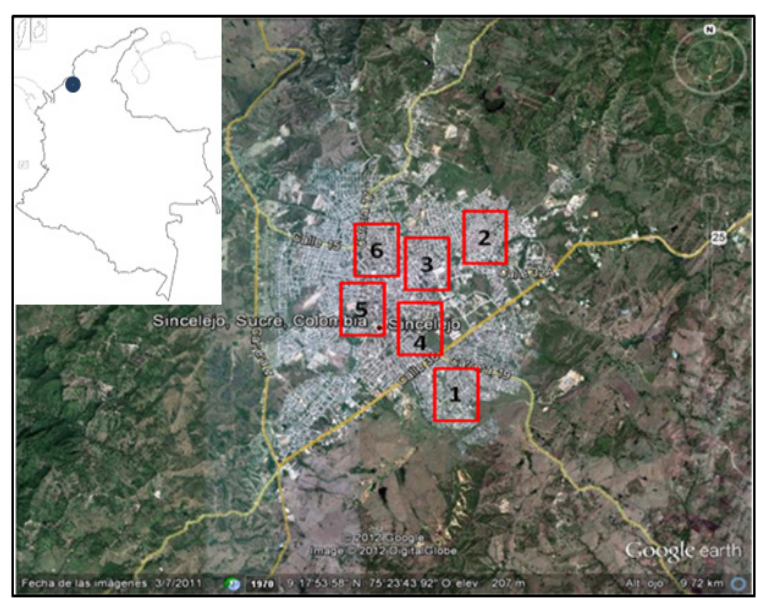

Figura 1. Áreas muestreadas en la ciudad de Sincelejo (Google Earth, versión libre. https://earth.google.com).

en los barrios de estrato 4 al 6 , que se denomina Grupo 2. La muestra representativa total calculada con base en el método de cálculo representativo para poblaciones finitas (ZAR, 1999) fue de 9 construcciones/grupo ( $p=$ 0,01 ; alfa $=99 \%$ y $d=8 \%$ ). Para los barrios, cuya suma total fue de 20 , se calculo, de igual forma una muestra representativa de 8 , equivalente a 4 por grupo $(p=0,01$; alfa $=99 \%$ y $d=10 \%$ ).

Evaluación de escombros: Las muestras se tomaron directamente del acopio temporal de cada obra de manera aleatoria utilizando un recipiente convencional de construcción y se pesaron mediante Báscula Bernalo ${ }^{\circledR}(150$ K y 50 gr de precisión); la clasificación se hizo manualmente separando los materiales encontrados en 6 grupos, cemento, geotextil, ladrillo, tubería, varilla y alambre. Seguidamente se pesó cada grupo de material para determinar el porcentaje de contenido en peso de cada uno de ellos en la muestra; se calculó la densidad de cada material de forma experimental y de esta manera con las cantidades en peso de cada uno se obtuvo el volumen respectivo. La densidad se calculó de forma experimental, seleccionando un recipiente de volumen conocido, en este caso se usaron cuñetes de pintura y se realizó una colecta selectiva de los residuos de los materiales considerados en el estudio de forma particular. Se taró el recipiente y por diferencia de peso entre el recipiente lleno y vacío se obtuvo el peso del residuo para cada material. Además, se midió la altura ocupada por el residuo en el recipiente con el fin de calcular el volumen (GIMÉNEZ et al., 2006).

Cálculo del Índice de generación de residuos o escombros (IGR): Adicionalmente se calculó el índice de generación de escombros (MERCANTE, 2006). El cual se estructura dividiendo el peso o el 
volumen de cada material residual por el área de la superficie construida. Este índice se utiliza para obtener cifras de generación que permiten comparar obras de características similares y para llevar un control de los residuos en la misma obra y poder evaluar temporalmente la generación de residuos.

Análisis de datos: Para el análisis estadístico de la parte relativa a los escombros producidos, además de utilizar las fórmulas antes enunciadas, los de los residuos clasificados fueron organizados en tablas, se aplicó correlación de Spearman y prueba de t simple; igualmente, en los casos en que fue necesario se calculó media, desvío estándar y porcentaje (ZAR, 1999).

\section{Resultados}

Análisis de la producción de escombros: En la Tabla 1 se muestran los datos medios del área de construcción de vivienda analizada en este estudio.

Tabla 1. Media de área $\left(\mathrm{m}^{2}\right)$ construida de acuerdo con el grupo.

\begin{tabular}{ccc}
\hline Estadístico & Grupo 1 & Grupo 2 \\
\hline Media & 70,78 & 151,59 \\
Máx & 115 & 210 \\
Min & 39 & 120 \\
DS & 22,945 & 20,844 \\
\hline
\end{tabular}

En la Tablas 2 y 3 se presentan los datos de peso de los residuos clasificados por grupo y por mes de estudio.

Tabla 2. Peso (kg) de los residuos clasificados por mes para el grupo 1.

\begin{tabular}{ccccccc}
\hline Residuos & Mes 1 & Mes 2 & Mes 3 & Mes 4 & Total & Promedio \\
\hline Cemento & 117,3 & 94,2 & 55,8 & 64,3 & 331,6 & 82,9 \\
Geotextil & 0,15 & 0,03 & 0 & 0 & 0,18 & 0,045 \\
Ladrillo & 29,3 & 90,5 & 115,7 & 54,9 & 290,4 & 72,6 \\
Tuberías & 117,8 & 118,2 & 69,5 & 95,4 & 400,9 & 100,225 \\
Varilla & 395,8 & 428 & 402,6 & 424,3 & 1650,7 & 412,675 \\
Alambre & 28,2 & 17,7 & 19,7 & 16,8 & 82,4 & 20,6 \\
\hline Total & 688,55 & 748,63 & 663,3 & 655,7 & 2756,18 & 689,045 \\
\hline
\end{tabular}

Tabla 3. Peso (kg) de los residuos clasificados por mes para el grupo 2.

\begin{tabular}{ccccccc}
\hline Residuos & Mes 1 & Mes 2 & Mes 3 & Mes 4 & Total & Promedio \\
\hline Cemento & 185,5 & 152,7 & 129,9 & 120,5 & 588,6 & 147,15 \\
Geotextil & 0,185 & 0,118 & 0 & 0 & 0,303 & 0,076 \\
Ladrillo & 0 & 163,6 & 174,5 & 145 & 483,1 & 120,775 \\
Tuberías & 195,8 & 202,3 & 159,8 & 189,6 & 747,5 & 186,875 \\
Varilla & 485,9 & 526,6 & 523,2 & 534 & 2069,7 & 517,425 \\
Alambre & 109 & 109,1 & 91,6 & 96,7 & 406,4 & 101,6 \\
\hline Total & 976,385 & 1154,418 & 1079 & 1085,8 & 4295,603 & 1073,901 \\
\hline
\end{tabular}

Al comprar estadísticamente mediante prueba de Spearman se tiene que existe diferencia significativa $(p<0.05)$ en cuanto al peso de los residuos clasificados totales por grupo $(\mathrm{N}=7$, Spearman 0,$9642 ; \mathrm{t}(\mathrm{N}$ 2)=8,1408; $p=0,0004)$.

En la Tablas 4 y 5 se presentan los cálculos de volumen de residuos clasificados por grupo.

Tabla 4. Volumen $\left(\mathrm{m}^{3}\right)$ los residuos clasificados para el grupo 1.

\begin{tabular}{cccc}
\hline Residuos & Peso Promedio & Densidad & Volumen \\
\hline Cemento & 82,90 & 1.100 & 0,0754 \\
Geotextil & 0,045 & 96,72 & 0,0005 \\
Ladrillo & 72,60 & 935,09 & 0,0776 \\
Tuberías & 100,22 & 168,21 & 0,5958 \\
Varilla & 412,67 & 876,50 & 0,4708 \\
Alambre & 20,60 & $1.177,52$ & 0,0175 \\
\hline & Total & & 1,2376 \\
\hline
\end{tabular}

Tabla 5. Volumen $\left(\mathrm{m}^{3}\right)$ de los residuos clasificados para el grupo 2.

\begin{tabular}{cccc}
\hline Residuos & Peso Promedio & Densidad & Volumen \\
\hline Cemento & 147,15 & 1.100 & 0,1338 \\
Geotextil & 0,075 & 96,72 & 0,0008 \\
Ladrillo & 120,77 & 935,09 & 0,1292 \\
Tuberías & 186,87 & 168,21 & 1,1109 \\
Varilla & 517,42 & 876,50 & 0,5903 \\
Alambre & 101,60 & $1.177,52$ & 0,0863 \\
\hline & Total & & 2,0512 \\
\hline
\end{tabular}

Al comprar estadísticamente mediante prueba de Spearman se tiene que existe diferencia significativa $(p<0.05)$ en cuanto al volumen de los residuos clasificados totales por grupo $(\mathrm{N}=7$, Spearman 0,9761 ; $t(N-2)=11,023 ; p=0,00003)$.

El porcentaje de los residuos clasificados por grupo se muestra en las Tablas 6 y 7 .

Tabla 6. Porcentaje del peso y el volumen de los residuos clasificados para el grupo 1.

\begin{tabular}{ccc}
\hline Residuos & Peso \% & Volumen \% \\
\hline Cemento & 12,03 & 6,09 \\
Geotextil & 0,01 & 0,04 \\
Ladrillo & 10,54 & 6,27 \\
Tuberías & 14,55 & 48,14 \\
Varilla & 59,89 & 38,04 \\
Alambre & 2,99 & 1,41 \\
\hline
\end{tabular}


Tabla 7. Porcentaje del peso y el volumen de los residuos clasificados para el grupo 2 .

\begin{tabular}{ccc}
\hline Residuos & Peso \% & Volumen \% \\
\hline Cemento & 13,70 & 6,52 \\
Geotextil & 0,01 & 0,04 \\
Ladrillo & 11,25 & 6,30 \\
Tuberías & 17,40 & 54,16 \\
Varilla & 48,18 & 28,78 \\
Alambre & 9,46 & 4,21 \\
\hline
\end{tabular}

Se tiene gráficamente una tendencia similar del comportamiento de los residuos clasificados por grupo, siendo evidente que el volumen, como es de esperarse para este tipo de actividad, sobrepasa la variable peso.

El cálculo del índice de generación de residuos (IGR) se presenta por discriminado por materiales clasificados y por grupo en las Tablas 8 y 9 .

Tabla 8. Calculo del índice de generación de residuos (IGR) para el grupo 1

\begin{tabular}{cccc}
\hline Residuo & Volumen & Área media & IGR $\left(\mathbf{m}^{3} / \mathbf{m}^{2}\right)$ \\
\hline Cemento & 0,0754 & 70,78 & 0,001 \\
Geotextil & 0,0005 & 70,78 & 0,000 \\
Ladrillo & 0,0776 & 70,78 & 0,001 \\
Tuberías & 0,595 & 70,78 & 0,008 \\
Varilla & 0,4708 & 70,78 & 0,007 \\
Alambre & 0,01749 & 70,78 & 0,000 \\
\hline Total & 1,2376 & 70,78 & 0,017 \\
\hline
\end{tabular}

Tabla 9. Calculo del índice de generación de residuos (IGR) para el grupo 2.

\begin{tabular}{cccc}
\hline Residuo & Volumen & Área media & IGR $\left(\mathbf{m}^{3} / \mathbf{m}^{2}\right)$ \\
\hline Cemento & 0,1338 & 151,59 & 0,001 \\
Geotextil & 0,0008 & 151,59 & 0,000 \\
Ladrillo & 0,1292 & 151,59 & 0,001 \\
Tuberías & 1,1109 & 151,59 & 0,007 \\
Varilla & 0,5903 & 151,59 & 0,004 \\
Alambre & 0,0862 & 151,59 & 0,001 \\
\hline Total & 2,0512 & 151,59 & 0,014 \\
\hline
\end{tabular}

Al comparar estadísticamente los índices de generación de residuos mediante prueba simple de $t$, no se determinan diferencias significativas $(p>0.05)$ (Tabla 10).
Tabla 10.Aplicación de prueba de t simple para determinar diferencias significativas para el IGR por grupo analizado.

\begin{tabular}{cccccccc}
\hline Grupo & Mean & Std.Dv. & $\mathbf{N}$ & Std.Err. & t-value & df & $\mathbf{P}$ \\
\hline $\mathbf{1}$ & 12,62937 & 35,70713 & 8 & 12,62438 & 1,000396 & 7 & 0,350438 \\
$\mathbf{2}$ & 12,62838 & 35,70753 & 8 & 12,62452 & 1,000306 & 7 & 0,350479
\end{tabular}

\section{Discusión}

El volumen de residuos producidos y su composición real, varía de un estrato a otro, como resultado del proceso socioeconómico en que cada uno está inmerso; igualmente pueden cambiar de una comunidad a otra, tal como se afirma en RECICLADO DE MATERIALES DE CONSTRUCCIÓN (2007) que lo atribuyen adicionalmenteala demografía histórica, al crecimientoy desarrollo actual de cada escenario analizado, lo cual concuerda con lo hallado en este estudio en donde se tuvo diferencias significativas al comparar los dos grupos de estratos respecto del peso y el volumen de los escombros. Sin embargo, la construcción de vivienda en Sincelejo, independientemente del estrato muestra características similares en cuanto al desecho de materiales, lo que queda establecido al observar la similitud en el índice de generación de residuos (IGR); existen diferencias entre estratos dados principalmente por el tamaño medio de las construcciones que en ellos se ejecutan y por las condiciones mismas de la actividad, en donde la exigencias de ley deficientes.

Por su parte el IGR puede variar con las características mismas de la construcción; según APL (2005) puede estar entre 0,050 $-0,178 \mathrm{~m}^{3} / \mathrm{m}^{2}$. MUÑOZ-ALVEAR et al. (2011) hallaron un IGR equivalente a 0,14 m³/m; D'APPOLONIA(1999) calculó un IGR de $0,150 \mathrm{~m}^{3} / \mathrm{m}^{2}$ y SERRANO (2009) de $0,120 \mathrm{~m}^{3} / \mathrm{m}^{2}$. Al tomar el rango antes señalado, los valores de IGR de este estudio: 0,017 $\mathrm{m}^{3} / \mathrm{m}^{2}$ (Grupo 1) y $0,014 \mathrm{~m}^{3} / \mathrm{m}^{2}$ (Grupo 2), son significativamente menoresy representarían entre $7,8 \%$ y $34 \%$ del rango establecido porAPL (2005).

El IGR y la cantidad de residuos estimada en la presente investigación se realizó solo para uso residencial y no se contemplaron criterios que pueden generar variaciones en los resultados obtenidos, como estimar el índice de generación teniendo en cuenta el uso comercial o identificar las variaciones en el índice conforme a los diferentes procesos constructivos industrializados que vienen ejecutando las obras de grandes superficiesoestimarlaconstrucción ilegal parasertenidaencuenta en la cantidad de RC generados por año en la ciudad, así como elaporte proveniente de las remodelaciones a los RC (AGUIRRE et al., 2005; HERNÁNDEZ, 2008).

La información analizada en el presente estudio muestra que no existe en el manejo de la construcción o reforma de vivienda urbana en la ciudad de Sincelejo una orientación hacia el paradigma de construcción sostenible, persiste el modelo de obra que construye y desecha, no existe un vertedero especializado ni grupo social organizado que reutilice adecuadamente los sobrantes aquí tipificados 


\section{Referencias}

AGUIRRE, C.; LATORRE, M.; BURBOA, R.; MONTECINOS, P. 2005. Diagnostico de la Generación de Residuos Sólidos de Construcción en Obras de Edificación en Altura en la Región Metropolitana. Revista de la Construcción 4 (2):38-46.

APL. 2005. Estudio de Impacto Ambiental y Económico del Acuerdo de Producción Limpia, de la construcción. Región Metropolitana, Chile.

ARENAS-CABELLO, F.J. 2007. El impacto ambiental en la Edificación. Criterios para una construcción sostenible. Edisofer. Madrid, España.

D’APPOLONIA, A. 1999. Informe preliminar Manejo integral de escombros y residuos de construcción.Banco Interamericano de Desarrollo. Washington, D.C.

GARCÍA-DONAS A.; AINCHIL-LAVÍN, J.P. 2009. Métodos de Comparación de efectos ambientales en el sector de la construcción. Revista de Planteamiento territorial y urbanismo 10. Disponible en: <985760 \%C1Garc\%EDa.pdf. Consultado: 9-11-2011.

GIMÉNEZ V.B.; CASTILLA CABANES, N.; CORTÉS LÓPEZ, J.M.; MARTÍNEZ ANTÓN, A.; PASTOR VILLA, R.M. 2006. Introducción al estudio de gestión de residuos de la construcción y demolición y estimación de cantidades generadas en obra. Departamento Construcciones Arquitectónicas. Centro ETS Arquitectura. Disponible en: http:/l riunet.upv.es/bitstream/handle/10251/7557/OA CALCULO RESIDUOS.pdf?sequence=1. Consultado. 06-06-2012. HERNÁNDEZ, B. 2008. Los residuos de la construcción. Técnica Industrial 273: 24 -30.

MERCANTE, I.T. 2006. Los residuos de Construcción en Mendoza. Estudio de Caso en Obra. Tesis de Maestría. Universidad Nacional de Cuyo. Argentina.

MUÑOZ-ALVEAR, E.; FABRES-JARA, A.; CÁRDENAS-RAMÍREZ. J.P. 2011. Residuos sólidos del proceso de construcción de viviendas en Chile - cuantificación, caracterización y establecimiento de indicadores. VI Encontro Nacional e IV Encontro Latino-americano sobre Edificações e Comunidades Sustentáveis. Elecs Vitória, Brasil.

RECICLADO DE MATERIALES DE CONSTRUCCIÓN. 2007. Disponible en: http://habitat.aq.upm.es/boletin/n2/ aconst1.html. Consultado: 20-04-2012.

SERRANO M.F., PÉREZ D. 2009. Propuesta de un programa de gestión integral de escombros. En: II Simposio I Iberoamericano de Ingeniería de Residuos. Universidad del Norte. Barranquilla.

SERRANO GUZMÁN, M.F.; PÉREZ RUÍZ, D.D. 2011. Concreto preparado con residuos industriales: resultado de alianza Empresa Universidad. Revista Educación en Ingeniería 11:1-11.

ZAR, J.H. 1999. Biostatistical Analysis, 4th. Ed. Prentice Hall, Upper Saddle River, NJ. USA. 